\title{
SOME LESS KNOWN WILD PLANT RESOURCES USED AS FOOD IN PUNE REGION OF MAHARASHTRA, INDIA
}

\author{
Samudra S. M. \\ K.G. Kataria College Daund, Pune-413801
}

\begin{abstract}
:
Pune region of Maharashtra is a treasury trove for wild edible plant resources. Detailed information of 156 species of wild plant species is enumerated in the present paper. In recent days, due to less awareness and anthropogenic pressure, many species of wild edibles are on the line of rarity. Therefore, there is urgent need to conserve and domesticate these valuable resources before whipping out. These wild edible germplasms are rich in neutraceuticles and phytochemicals. Extensive explorations were made for the documentation of ethnobotanical knowledge from the tribals, villagers, elder persons, residents etc.
\end{abstract}

Keywords-Wild edibles, ethnobotany

\section{INTRODUCTION:}

Wild edible plants constitute an important source of dietary habit of tribals and villagers living in and around the forests. In the modern agriculture, they can be used for the development of varieties. The wild genetic resources are the building blocks of new varieties which are eroding fastly due to spread of high yielding varieties, shrinking of natural habitats, rapid increase in urbanization and environmental threats, anthropogenic interference etc. Out of 17,000 documented species, one third species are of economic use (Chandel, 1997). The documentation and inventory preparation of wild edibles are essential for investigation of new crop of edible plants in the present era.

Efforts have been made by Billore, 1972; Cook, T. 1958 Repr. ed.; Datar, 1975; Kulkarni and Kumbhojkar, 1993; Kumbhojkar and Vartak 1988; Mokat, 2005; Upadhye et al. 1986; Vartak and.Gadgil, 1959; Vartak and Gadgil, 1980 for documentation of wild edible plants, their ethnobotany and uses in Maharashtra in general and Western Ghats in particular. In India, about 800 species of wild edible plants consumed as food plants by the tribal peoples (Jain et al. 1977; Arora 1978; Jain and Dam, 1979; Arora, 1981; Singh et al.1996) reported wild edibles from different parts of the country.

\section{MATERIALS AND METHODS:}

Several explorations were conducted during 2008-2012 in Pune region of Western Ghat's in districts viz., Pune, Satara and Kolhapur. The details viz. local name, part used for consumption and mode of utilization etc. was documented. The wild edible plants were collected from different places and identified with the help of local floras Singh et al. 2000 \& 2001; Sharma et. al 1996; Cook, 1958, and also confirmed from Botanical Survey of India, Western Circle, Pune.

Table 1.- The list of plant documented at the time of study

\begin{tabular}{|l|l|l|l|l|l|}
\hline $\begin{array}{l}\text { Sr.N } \\
\text {.. }\end{array}$ & Botanical Name of Plants & Family & Local Name & Part Used & Uses \\
\hline 1. & Abrus precatorius L. & Fabacae & Gunj & Leaves & Fresh and dried leaves are eaten. \\
\hline 2. & Aegle marmelos (L). Correa & Rutaceae & Bael & Fruits & Fruit pulp is eaten. \\
\hline 3. & Agave Americana L. & Agavaceae & Ghypat & Flower & Flower are fried, eaten as a vegetable \\
\hline 4. & Amaranthus spinosus L. & Amaranthacea & Katemath & Leaves & Used as vegetable. \\
\hline 5. & Amaranthus tricolor L. & Amaranthacea & Rajgira & Leaves & Used as vegetable. \\
\hline 6. & Amaranthus tricolor L. & Amaranthacea & Tanduja & Leaves & Used as vegetable. \\
\hline 7. & Amranthus viridis L. & Amaranthacea & Math & Leaves & Used as vegetable. \\
\hline 8. & $\begin{array}{l}\text { Amorphophallus companulatus } \\
\text { Decene) }\end{array}$ & Araceae & Suran & Tuber & Tuber used as vegetable. \\
\hline 9. & $\begin{array}{l}\text { Ampelocissus latifolia } \text { (Roxb.) } \\
\text { Planch }\end{array}$ & Vitaceae & Nadena & Fruits & Tribal and local people eat ripe fruit. \\
\hline 10. & Annona reticulate L. & Annonaceae & Ramphal & Fruit & Ripen fruit are eaten. \\
\hline 11. & Annon squamosa L. & Annonaceae & Sitaphal & Fruit & Ripen fruit are eaten. \\
\hline 12. & Areca catechu L. & Areacea & Supari & Nut & Nut are eaten, often eaten with betel \\
\hline 13. & Artocarpus heterophyllus Lam. & Moraceae & Phanas & Fruit & Fruit and seeds are edible. \\
\hline
\end{tabular}




\begin{tabular}{|c|c|c|c|c|c|}
\hline 14. & Asparagus racemosus Willd. & Liliaceae & Shatavari & Tuberous root & Root powder taken with milk. \\
\hline 15. & Averrhoa bilimbii $L$. & Averrhoaceae & Bilimbi & Fruit & $\begin{array}{l}\text { Fruits are edible, pickles prepared from } \\
\text { fruit. }\end{array}$ \\
\hline 16. & $\begin{array}{l}\text { Bambusa arundianacea (Retz.) } \\
\text { Willd. }\end{array}$ & Poaceae & Kalak & Rhizome & New shoot used as vegetable. \\
\hline 17. & Basella rubra L. & Basellaceae & Mayalu & Leaves & Leaves used for vegetable. \\
\hline 18. & Benincasa hispida (Thunb.) Cogn. & Cucurbitaceae & Kohala & Fruit & Fruit used as vegetable. \\
\hline 19. & Borassus flabellifer $L$. & Arecaceae & Tad & Fruit & Fruits are edible. \\
\hline 20. & $\begin{array}{l}\text { Brassica juncea (L.) Czem. \& } \\
\text { Coss. }\end{array}$ & Brassicaceae & Mohair & Leaves & Used as vegetable. \\
\hline 21. & Bridelia retusa (L.) & Ephorbiaceae & Asana & Fruit & Ripened fruits are edible. \\
\hline 22. & Bridelia squamosa (Lam.) Gehm & Ephorbiaceae & Asana & Fruit & Ripened fruits are edible. \\
\hline 23. & Cajanus cajan (L.) Millsp. & Fabaceae & Tur & Seed/pod & Used as vegetable. \\
\hline 24. & Canavalia ensiformis (L.) DC & Fabaceae & Abai & Fruit & Used as vegetable. \\
\hline 25. & Capparis moonii Wight. & Capparaceae & Waghati & Fruit & $\begin{array}{l}\text { Ripe fruit are edible. Raw fruit are } \\
\text { cooked and used as vegetable. }\end{array}$ \\
\hline 26. & Capparis sepiaria L. & Capparaceae & Waghati & Fruit & Ripened fruit are edible. \\
\hline 27. & Capparis zeylanica $L$. & Capparaceae & Waghati & Fruit & Fruits are edible. \\
\hline 28. & Carissa congesta Wight. & Apocynaceae & Karvand & Fruit & $\begin{array}{l}\text { Unripe fruits are used for preparation } \\
\text { of pickles and ripened fruits for making }\end{array}$ \\
\hline 29. & Carica papaya $L$. & Caricaceae & Papai & Fruit & Fruit are eaten. \\
\hline 30. & Caryota urens $L$. & Araceae & Bherli-mad & Seed & Seeds are eaten. \\
\hline 31. & Cassia fistula $L$. & $\begin{array}{l}\text { Caesalpiniacea } \\
\text { e }\end{array}$ & Bahava & Fruit pulp & Pulp of the pod is eaten. \\
\hline 32. & Cassia occidentalis $L$. & $\begin{array}{l}\text { Caesalpiniacea } \\
\text { e }\end{array}$ & Tarvad & Leaves & Used as vegetable. \\
\hline 33. & Cassia pumila Lam. & $\begin{array}{l}\text { Caesalpiniacea } \\
\text { e }\end{array}$ & Chinchani & Seed & Seeds are eaten. \\
\hline 34. & Cassia tora $L$. & $\begin{array}{l}\text { Caesalpiniacea } \\
\text { e }\end{array}$ & $\begin{array}{l}\text { Takla- } \\
\text { tarvad }\end{array}$ & Leaves & $\begin{array}{l}\text { Leaves and young shoots used as } \\
\text { vegetable. }\end{array}$ \\
\hline 35. & Cayratia camosa Gagnep. & Vitaceae & $\begin{array}{l}\text { Dukarvel } \\
\text { ambat }\end{array}$ & $\begin{array}{l}\text { Leaves, stem and } \\
\text { fruit }\end{array}$ & $\begin{array}{l}\text { Boiled leaves and stem are used for } \\
\text { vegetable. Ripen fruit eaten. }\end{array}$ \\
\hline 36. & Celosia argentea $L$. & $\begin{array}{l}\text { Amaranthacea } \\
\mathrm{e}\end{array}$ & Kardu & Leaves & Leaves used as vegetable. \\
\hline 37. & Ceropegia bulbosa Roxb. & $\begin{array}{l}\text { Asclepiadacea } \\
\mathrm{e}\end{array}$ & $\begin{array}{l}\text { Khapparka } \\
d u\end{array}$ & Tuber & Tubers are edible. \\
\hline 38. & $\begin{array}{l}\text { Chlorophytum tuberosum (Roxb.) } \\
\text { Baker }\end{array}$ & Liliaceae & Phodshi & Leaves, Tuber & $\begin{array}{l}\text { Leaves are used as vegetable. Tuber are } \\
\text { eaten. }\end{array}$ \\
\hline 39. & Cissus adnata Roxb. & Vitaceae & Naden & Leaves & Cooked leaves are eaten. \\
\hline 40. & Cissus discolor Blume & Vitaceae & Telicha vel & Fruit & Fruits are eaten. \\
\hline 41. & Cissus quadrangularis $L$. & Vitaceae & Kandvel & Stem & Stem are eaten in cirries. \\
\hline 42. & Cissus rependa Vahl. & Vitaceae & Gendal & Stem & $\begin{array}{l}\text { Juice obtained from the vines is } \\
\text { drinkable. }\end{array}$ \\
\hline 43. & Cissus repens Lam. & Vitaceae & Basil & Leaves & Leaves are cooked and eaten. \\
\hline \multirow[t]{17}{*}{44.} & Citrullus colocynthis (L.) Schrad & Cucurbitaceae & $\begin{array}{l}\text { Kadu- } \\
\text { indrayan }\end{array}$ & Grains & seeds are edible. \\
\hline & $\begin{array}{l}\text { Citrullus lanatus (Thunb.) Mats \& } \\
\text { Nakai }\end{array}$ & Cucurbitaceae & Tarbuj & Fruit & Fruits are edible \\
\hline & Citrus medica L. & Rutaceae & Mahalungi & Fruit & $\begin{array}{l}\text { Fruits are eaten and also used for } \\
\text { making iuice. }\end{array}$ \\
\hline & Clerodendrum serratum (L.) Moon & Verbenaceae & Bharangi & Leaves & Leaves are used as a vegetable \\
\hline & Coccinia grandis (L.) Voigt & Cucurbitaceae & Tondli & Fruit & Fruits are edible and used as vegetable. \\
\hline & Cocos nucifera L. & Arecaceae & Narel & Fruit & Leaves are used for thatching. \\
\hline & Coix lachryma-jobi L. & Poaceae & Kusav & Seeds & $\begin{array}{l}\text { Seeds are used for making necklace. } \\
\text { Leaves are used as a fodder for live }\end{array}$ \\
\hline & Colocasica esculenta (L.) Schott & Araceae & $\begin{array}{l}\text { Bhajiche } \\
\text { alu }\end{array}$ & Leaves & Leaves used as vegetable \\
\hline & Commelina benghalensis L. & $\begin{array}{l}\text { Commelinacea } \\
\mathrm{e}\end{array}$ & Kena & Leaves & Leaves used as vegetable \\
\hline & Cordia dichotoma Forst & Boraginaceae & Bhokar & Fruit & Fruits are edible \\
\hline & Coriandrum sativum $\mathrm{L}$. & Apiaceae & Kothimbir & Fruit & Fruit used as condiment \\
\hline & Costus speciosus (Koen) J.E.Sm. & Costaceae & Coast & Leaves & Leaves used as vegetable \\
\hline & Cucumis callosus (Roettl.) Cogn. & Cucurbitaceae & - & Fruit & Fruits used as vegetable \\
\hline & Cucumis melo L. & Cucurbitaceae & Karit & Fruit & Fruits are edible \\
\hline & Cucurbita maxima Duch.ex.Lam & Cucurbitaceae & $\begin{array}{l}\text { Tambda } \\
\text { Bhopala }\end{array}$ & Fruit & Fruits used as vegetable \\
\hline & Cucurbita pepo L. & Cucurbitaceae & $\begin{array}{l}\text { Kashi- } \\
\text { Bhopala }\end{array}$ & Fruit & Fruits used as vegetable \\
\hline & Curcuma amada Roxb. & Zinziberaceae & Ran-Halad & Tuber & $\begin{array}{l}\text { Tuberous root used for preparation of } \\
\text { pickles }\end{array}$ \\
\hline
\end{tabular}




\begin{tabular}{|c|c|c|c|c|c|}
\hline & Curcuma aromatica Salisb & Zinziberaceae & $\begin{array}{l}\text { Amba- } \\
\text { Halad }\end{array}$ & Tuber & $\begin{array}{l}\text { Tuberous root used for preparation of } \\
\text { pickles }\end{array}$ \\
\hline & $\begin{array}{l}\text { Cyamopsis tetragonolobus (L.) } \\
\text { Taub }\end{array}$ & Fabaceae & Gawar & Fruit & Fruits used as vegetable \\
\hline & Dendrophthoe falcata (L.f.) & Loranthaceae & Bandgul & Fruit & Fruits are eaten. \\
\hline & Dillenia indica $\mathrm{L}$. & Dilleniaceae & $\begin{array}{l}\text { Mota- } \\
\text { Karmal }\end{array}$ & Fruit & Sepals are eaten \\
\hline & Dioscorea alata $L$. & Dioscoreaceae & Ghorkhand & Tuber & Tubers used as vegetable \\
\hline & Dioscorea bulbifera $L$. & Dioscoreaceae & Karanda & Tuber & Tubers used as vegetable \\
\hline & $\begin{array}{l}\text { Dioscorea esculenta (Lour.) } \\
\text { Burkill }\end{array}$ & Dioscoreaceae & Kangar & Tuber & Tubers used as vegetable \\
\hline & Dioscorea pentaphylla L. & Dioscoreaceae & Shendvel & Inflorescence & Flowers used as vegetable. \\
\hline & Diospyros melanoxylon Roxb. & Ebenaceae & Tendu & Fruit & Fruits are edible \\
\hline & $\begin{array}{l}\text { Embelia basaal (R \& S.) A. DC. } \\
\text { Trans. Linn }\end{array}$ & Myrsinaceae & Ambati & Fruit & Fruits are edible \\
\hline & Emblica officinalis Gaertn & Euphorbiaceae & Avala & Fruit & Fruits are edible \\
\hline & Ensete superbum (Roxb.) Cheesm & Musaceae & Rankel & Fruit & Fruits are edible \\
\hline & Ficus benghalensis $L$. & Moraceae & Wad & Fruits & Fruits are edible. \\
\hline & Ficus racemosa $L$. & Moraceae & Umbar & Fruits & Fruits are edible. \\
\hline & Flacortia Montana Grah & Flacourtiaceae & Attak & Fruit & Ripe fruits are edible \\
\hline & $\begin{array}{l}\text { Garcinia indica (Du Petit-Thou.) } \\
\text { Choisv }\end{array}$ & Clusiaceae & $\begin{array}{l}\text { Amruta, } \\
\text { Kokum }\end{array}$ & Fruit & $\begin{array}{l}\text { Ripe fruits are edible. Fruits used for } \\
\text { preparation of iuice and svrup. The }\end{array}$ \\
\hline & $\begin{array}{l}\text { Garcinia xanthochymus Hook. f. } \\
\text { ex T }\end{array}$ & Clusiaceae & $\begin{array}{l}\text { Kadu } \\
\text { Kokum }\end{array}$ & Fruit & Ripe fruits are edible \\
\hline & Garuga pinnata Roxb. & Burseraceae & Kakad & Fruit & Fruits are edible \\
\hline & Grewia asiatica $L$. & Tiliaceae & Phalsi & Fruit & Ripe fruits are edible \\
\hline & Grewia tillifolia Vahl & Tiliaceae & Dhaman & Fruit & Ripe fruits are edible \\
\hline & $\begin{array}{l}\text { Habenaria grandifloriformis Blatt. } \\
\& \mathrm{McC} \text {. }\end{array}$ & Orchidaceae & Hilkurkand & Fruit & Bulbs are edible \\
\hline & Hibiscus sabdariffa L. & Malvaceae & Ambadi & Leaves & Leaves used as a fodder. \\
\hline & $\begin{array}{l}\text { Holarrhena pubescens (Buch.- } \\
\text { Ham.) Wall. ex G. Don }\end{array}$ & Apocynaceae & $\begin{array}{l}\text { Pandhara } \\
\text { Kuda }\end{array}$ & Pod & Young pods used as a vegetable \\
\hline & $\begin{array}{l}\text { Hyphaene dichotoma (White) } \\
\text { Furtado }\end{array}$ & Arecaceae & Ravan-Tad & Fruit & Fruits are edible \\
\hline & Indigofera cordifolia Heyne & Fabaceae & Bechka & Seeds & Used like cereal. \\
\hline & Indigofera linifolia retz. & Fabaceae & $\begin{array}{l}\text { Phandarph } \\
\text { alli }\end{array}$ & Grains & Used like cereal. \\
\hline 87. & Ipomoea aquatica Forssk. & $\begin{array}{l}\text { Convolvulacea } \\
\text { e }\end{array}$ & $\begin{array}{l}\text { Nalichi } \\
\text { bhaii }\end{array}$ & Leaves & Used as leafy vegetable \\
\hline & Ixora brachiata Roxb. & Rubiaceae & Lokhandi & Fruit & Fruits are edible \\
\hline & Ixora coccinea $L$. & Rubiaceae & Devara & Fruit & Fruits are edible \\
\hline & Lantana camara $L$ & Verbenanceae & Ghaneri & Fruit, Leaves & $\begin{array}{l}\text { Fruits are edible. Leaves are eaten with } \\
\text { sandal bark as like beetal leaf. }\end{array}$ \\
\hline & Leea indica (Burm.f.) Merr. & Leeaceae & Dinda & Leaves & Used as leafy vegetable. \\
\hline & Limonia acidissima $L$. & Rutaceae & Kavath & Fruit & Fruits are edible \\
\hline & Luffa acutangula (L.) Roxb. & Cucurbitaceae & Ran-dodka & Fruit & Fruits used as vegetable \\
\hline & $\begin{array}{l}\text { Madhuca longifolia (Koen.) Mac } \\
\text { Bride }\end{array}$ & Sapotaceae & Moha & Fruit & Fruits are edible \\
\hline & $\begin{array}{l}\text { Mammea suriga (Buch.-Ham. ex } \\
\text { Roxb.) Kosterm. }\end{array}$ & Clusiaceae & Surangi & Fruit & Ripe fruits are edible \\
\hline & Mangifera indica $L$ & Anacardiaceae & $A m b a$ & Fruit & Fruits and endosperm are edible \\
\hline & Manilkara hexandra (Roxb.) Dub & Sapotaceae & Khirni & Fruit & Fruits are edible \\
\hline & Manilkara zapota (L.) van Royen & Sapotaceae & Chikku & Fruit & Fruits are edible \\
\hline & $\begin{array}{l}\text { Marsdenia tenacissima (Roxb.) } \\
\text { Moon }\end{array}$ & $\begin{array}{l}\text { Asclepiadacea } \\
\mathrm{e}\end{array}$ & - & Thalamus & Thalamus is edible. \\
\hline & $\begin{array}{l}\text { Memecylon umbellatum Burm. f. } \\
\text { Fl }\end{array}$ & $\begin{array}{l}\text { Melastomatace } \\
\text { ae }\end{array}$ & Anjan & Fruit & $\begin{array}{l}\text { Ripe fruits are edible; Dye is prepared } \\
\text { from leaves and fruits. }\end{array}$ \\
\hline & Meyna laxiflora Robyns & Rubiaceae & Alu & Fruit & Mature fruits are edible \\
\hline & Mimusopa elengi L. & Sapotaceae & Bakul & Fruit & Fruits are edible \\
\hline & Momordica charantia $L$. & Cucurbitaceae & Karle & Fruit & Fruits used as vegetable \\
\hline & Momordica dioica Roxb. ex Willd. & Cucurbitaceae & Kartoli & Fruit & Fruits used as vegetable \\
\hline & Moringa concanensis Nimmo. & Moringaceae & Kadushegat & Leaves, Fruit & $\begin{array}{l}\text { Leaves and fruits are cooked and used } \\
\text { as vegetable }\end{array}$ \\
\hline & Moringa oleifera Lam. & Moringaceae & Shevga & $\begin{array}{l}\text { Leaves, Fruit, } \\
\text { Flowers }\end{array}$ & $\begin{array}{l}\text { Leaves, fruits and flowers are cooked } \\
\text { and used as a vegetable }\end{array}$ \\
\hline & Musa paradisiaca $L$. & Musaceae & Kela & Fruit & $\begin{array}{l}\text { Fruits are edible. Flowers used as } \\
\text { vegetable }\end{array}$ \\
\hline & Myristica fragrans Houtt & Myristicaceae & Jayphal & $\begin{array}{l}\text { Seed, seed coat, } \\
\text { Epicarp }\end{array}$ & Used as a spice and condiments \\
\hline
\end{tabular}




\begin{tabular}{|c|c|c|c|c|}
\hline $\begin{array}{l}\text { Neolamarckia cadamba (Roxb.) } \\
\text { Bosser }\end{array}$ & Rubiaceae & Kadam & Flowers & The flower heads are edible \\
\hline Nymphaea pubescens Willd & Nymphaceae & Kamal & Root stock & Used as vegetable. \\
\hline Ocimum gratissimum $L$. & Lamiaceae & Tulsi & Leaves & Leaves are eaten. \\
\hline Ocimum gratissimum $L$. & Lamiaceae & Sabja & Leaves & Leaves used as vegetable. \\
\hline Ocimum tenuiflorum $L$. & Lamiaceae & Tulas & Leaves & Leaves are edible. \\
\hline Oroxylum indicum (L.) Vent. Dec & Bignoniaceae & Tetu & Seeds & Seeds are edible. \\
\hline Oryza sativa $L$. & Poaceae & Bhat & Grain & Grains are used as a cereal. \\
\hline Oxalis corniculata $L$. & Oxalidaceae & Ambusi & Leaves & Used as leafy vegetable. \\
\hline Panicum miliaceum L. & Poaceae & Varai & Grain & Seeds used as a cereal. \\
\hline Paspalum scrobiculatum L. & Poaceae & Harik & Grain & Used like cereals. \\
\hline Pavetta indica $L$. & Rubiaceae & Papat & Flowers & Used as vegetable. \\
\hline Pergularia daemia (Forssk.) Choiv & $\begin{array}{l}\text { Asclepiadacea } \\
\mathrm{e}\end{array}$ & Utran & $\begin{array}{l}\text { Flower, } \\
\text { Inflorescence }\end{array}$ & Used as vegetable. \\
\hline Phaseolus sublobatus Roxb. & Fabaceae & Makani & Seed & Used like cereals. \\
\hline Physalis minima $L$. & Solanaceae & Chirboti & Fruit & Fruits and Thallamus are edible \\
\hline Plumbago zeylanica $L$. & $\begin{array}{l}\text { Plumbaginace } \\
\text { ae }\end{array}$ & Chitrak & Leaves & Leaves used as vegetable \\
\hline Portulaca oleracea $L$. & Portulaceaeae & Ghol & Leaves, shoots & Leaves used as vegetable \\
\hline Portulaca quadrifida $L$. & Portulaceaeae & $\begin{array}{l}\text { Chival, } \\
\text { Chota Ghol }\end{array}$ & Whole plant & Whole plant used as a vegetable. \\
\hline Psidium guajava L. & Myrtaceae & Peru, Jamb & Fruit & Fruits are edible \\
\hline Puraria tuberosa DC. & Fabaceae & Pithana & Root & Boiled or raw tuberous roots are eaten. \\
\hline Raphanus sativus $L$. & Brassicaceae & Mula & Root & Used as vegetable \\
\hline $\begin{array}{l}\text { Remusatia vivipara (Roxb.) } \\
\text { Schott. \& Endl }\end{array}$ & Araceae & Mayajal & Leaf & Leaves used as vegetable \\
\hline Ricinus communis $L$. & Euphorbiaceae & Erand & Seed & Seeds are edible \\
\hline Santalum album $L$. & Santalaceae & Chandan & Fruit & Fruits are edible \\
\hline Schleichera oleosa (Lour.) Oken & Sapindaceae & Kusum & Fruit & The ripe fruits are edible \\
\hline Semecarpus anacardium L.f. & $\begin{array}{l}\text { Anancardiacea } \\
\mathrm{e}\end{array}$ & Bibba & Fruit & Decoction of fruits used for gargling \\
\hline Sesamum orientale $L$. & Pedaliaceae & Rantil & Seeds & Seeds are edible \\
\hline Setaria glauca Beauv. & Poaceae & Kolara & Seeds & Used as a cereal. \\
\hline Smithea sensitiva Ait & Fabaceae & Kevla & Whole plant & Used as leafy vegetable \\
\hline Solanum anguivi Lam. & Solanaceae & Dorli & Leaves, Fruit & Fruits are used as a vegetable \\
\hline Solanum melongena $L$. & Solanaceae & Wange & Fruit & Used as vegetable \\
\hline Solanum tuberosum $L$. & Solanaceae & Batata & Tuber & Used as vegetable \\
\hline $\begin{array}{l}\text { Solena amplexicaulis (Lam.) } \\
\text { Gandhi }\end{array}$ & Cucurbitaceae & Gomati & Fruit & Fruits are edible \\
\hline Spondias pinnata (L.f.) Kurz & Anacardiaceae & Ambada & Fruit & Fruits are edible \\
\hline Sterculia urens Roxb. & Sterculiaceae & Kandol & Kernels & Kernels are edible \\
\hline Syzygium cumini (L.) Skeels & Myrtaceae & Jambhul & Fruit & Ripe fruits are edible \\
\hline Tagetes erecta $L$. & Asteraceae & Zendu & Flower & Flowers are edible \\
\hline Tamarindus indica $L$. & $\begin{array}{l}\text { Caesalpiniacea } \\
\mathrm{e}^{\text {en }}\end{array}$ & Chinch & Seed, Pulp & Both ripe and unripe fruits are edible \\
\hline $\begin{array}{l}\text { Terminalia bellirica (Gaertn.) } \\
\text { Roxb. }\end{array}$ & Combretaceae & Behda & Kernels & Mesocarp and seeds are edible \\
\hline $\begin{array}{l}\text { Tetrastigma lanceolarium (Roxb.) } \\
\text { Planch. }\end{array}$ & Vitaceae & Choral vel & Fruits & Eaten after cooking. \\
\hline Tricholepis amplexicaulis Clarke & Asteraceae & Dahan & Leaves & Used as leafy vegetable \\
\hline Trichosanthes dioica Roxb. & Cucurbitaceae & Padval & Fruit & Used as vegetable \\
\hline Vigna radiata (L.) Wilczeck & Fabaceae & Udid & Seed & Seeds are used for making fry. \\
\hline Vigna vexillata (L.) A.Rich. & Fabaceae & Halunda & Root & Raw roots are eaten. \\
\hline Woodfordia fruticosa (L.) Kurz & Lythraceae & Dhayati & Flower & Dried flowers are eaten \\
\hline Xanthosoma sagittifolium & Araceae & Vadiche alu & Leaves & Used as leafy vegetable \\
\hline Zingiber officinale Rosc. & Zingiberaceae & Ale & Rhizome & Rhizome are edible \\
\hline Ziziphus jujuba Mill & Rhamnaceae & Bor & Fruit & The ripe fruits are edible \\
\hline Ziziphus rugosa Lam. & Rhamnaceae & Toran & Fruit & Fruits are edible \\
\hline
\end{tabular}




\section{RESULTS AND DISCUSSION:}

The 156 wild plants species were recorded and enumerated. Leaves of 36 plant species are used as a vegetable and fruits of 79 species, inflorescence of 2 species, 10 tuberous species, 13 seeds, 05 grains, 2 rhizome, 5 roots, 1 whole plant, thalamus of 1 species, and seed aril of one species are utilized and consumed. Tribals, villagers, tourists etc. are utilizing these wild edibles as vegetable, for making different products or eaten as a raw. Germplasms of many wild edibles viz. Colocasias, Dioscoreas, Ipomea, Momordica, Xanthosoma, Cucumis, Solanum, Cajans, Vigna, Oryza, Eleusine, and millets etc. have been conserved and maintained by tribals and villagers in and around their houses and their agricultural fields. They are selecting the best one through mass selection and cultivating near the houses. It is also observed that, tribals exchanging the germplasm resources for sowing, consumption, for offering to god or goddess etc. There are limited reports regarding wild edible plants. Kulkarni and Kumbhojkar (1993) reported 40 kitchen garden plants. There is need to investigate phytochemicals, neutraceuticles etc. for the betterment of society health.

\section{REFERENCES:}

Arora, R. K. (1981). Native Food plants of the North eastern Tribes in S.K. Jain Ed. Glimpses of Indian Ethnobotany. Oxford \& IBH Publishing Co. New Delhi. Pp.91-106.

Billore, K. V. (1972). Studies on the vegetation and flora of Thana district, MS. Vol.I \&II. Thesis submitted to Vikram University, Ujjain (MP) for the degree of Doctor of Philosophy in Botany.

Bothmer, R. Von and O. Seberg (1995). Strategies for collection of wild species (Eds Guarino L., V. Raantha Rao and R. Reid.) Collecting Plant Genetic Diversity. Technical guidelines, CAB international, UK. pp. 93-112.

Chandel, K.P.s. (1997). Forward in Crop Improvement Through Plant Genetic Resources (Eds. D. P. Patel, V. D. Verma, T. R. Loknathan, M. N. Kapoor and K. P. S. Chandel) Akola.

Cook, T. (1958 Repr. ed.). The Flora of the Presidency of Bombay. BSI, Culcutta.

Datar, Rekha and V. D. Vartak (1975). Enumeration of wild edible plants from Karnala bird sanctuary, MS. Biovigyanam 1:123-129.

Human Z., F. det Puente and C. Arbizu, (1995). Collecting vegetatively propagated crops
International Journal of Indigenous Medicinal Plants, ISSN: 2051-4263, Vol.47, Issue. 11

(especially roots and tubers). Collecting plant genetic diversity: Technical guidelines, CAB international, U. K. pp 457-466.

Jain, S. K. \& A. K. Hajra \& R. Shanpru (1977). A survey of edible plants in bazaars of Meghalaya. Bull. Meghalaya Sc.Soc. 2:29-34.

Jain, S. K. \& N. Dam (1979). Some ethnobotanical note from north eastern India. Econ. Bot. 33(1):52-56.

Kulkarni, D.K. and M.S. Kumbhojkar (1993). Kitchen garden plants of Mahadeokoli tribe in Maharashtra. Etnhobotany.5:119-127.

Kumbhojkar, M. S. \& V. D. Vartak (1988). Ethnobotanical studies on wild edible grapes from sacred groves in Western Maharashtra. J.Econ. Tax.Bot. Vol. 12(2):257-263.

Mokat, D. N. (2005). Ethno-medico-botanical survey of Thane and Ratnagiri districts of Maharashtra with respect to its indigenous knowledge with an aid of Pharmacognosy. Thesis submitted to Pune university, Pune (MS) for the degree of doctor of Philosophy in Botany.

Mokat, D. N. and B. B. Jadhav (2003) Species diversity and germplasm collection of medicinal plants from Western Ghats of Maharashtra. Ind. J. Plant Genetic Resour. Vol. 16. No. 2 pp.126132.

Mokat, D. N. and S. S. Deokule (2006) Ethnomedico-botanical survey of Ratnagiri district of Maharashtra. JETB. Vol. No. 30 Suppl. PP. 140144.

Sharma, B. D., Karthikeyan, S. and Singh, N. P. (1996). Flora of Maharashtra state Monocotyledons. BSI. Calcutta.

Singh, H.B. \& R.K. Arora (1978). Wild edible plants of India, ICAR, New Delhi.

Singh, N. P., Lakshinarasimshan, P., Karthikeyan, S. and Prasanna 2001. Flora of Maharashtra state Dicotyledones, Vol. II. BSI, Calcutta.

Singh, N. P. and Karthikeyan, S. 2000. Flora of Maharashtra state. Dicotyledones, Vol. I BSI, Calcutta.

Singh, Umrao, A .M. Wadhwani and B. M. Johri (1996). Dictionary of Economic Plants in India. ICAR, New Delhi.

Upadhye, A., M. S. Kumbhojkar \& V. D. Vartak (1986). Observation on wild plants used in the rural area of the Kolhapur district. Ancient Sci. Life. 6(2): 119-121.

Vartak, V. D. and M. Gadgil (1959). Some edible wild plants from the hilly regions of Poona district, Bombay state. J.Bombay.Nat.Hist.Soc. 56:8-25. 
Vartak, V. D. and Madhav Gadgil (1980). Studies in Ethnobotany-A new vista in botanical sciences. Biovigyanam. 6:151-156. (C) 